\title{
Preuve ou orthodoxie?
}

\section{Anna Sax}

Lic. oec. publ., MHA, membre de la rédaction

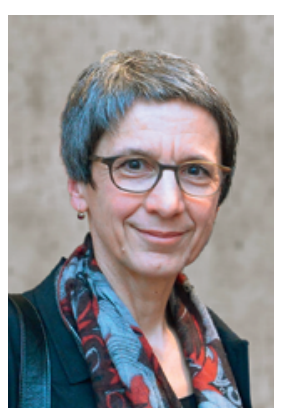

«Que fait la Commission de la concurrence?», demande Stefan Felder, professeur en économie de la santé, qui déplorait, dans un article récemment publié, que les hôpitaux publics aient «une position dominante» dans beaucoup de cantons. Comme nombre de ses collègues partisans de l'orthodoxie libérale, il pense qu'une concurrence libre entre les hôpitaux ferait baisser les coûts et améliorerait la qualité. Il prend pour référence une étude américaine vieille de 17 ans. «L'indice de dérégulation», déjà présenté une fois et mis à jour entretemps par le bureau de conseil d'Olten, Polynomics, enfonce le clou et note les cantons en fonction de la distance qu'ils entretiennent avec les soins hospitaliers. L'avantage du fédéralisme serait de pouvoir observer et comparer les effets des différentes politiques. Mais les adeptes de cette orthodoxie de la dérégulation doivent encore nous prouver quels sont les avantages dont bénéficient les patients, les payeurs de primes et les contribuables dans les cantons qui suivent leurs recommandations. Ni le «Monitoring de l'évolution des coûts de l'assurance maladie» (de l'OFSP), ni les tests aléatoires faits sur les différents portails de comparaison de la qualité ne permettent de conclure qu'une plus grande concurrence entre les hôpitaux diminue les coûts, améliore la satisfaction des patients et aboutit à une meilleure qualité.

On ne voit donc pas pourquoi les cantons devraient renoncer à gérer leurs propres hôpitaux. A qui cela va-t-il profiter, si par exemple l'hôpital cantonal de Winterthour est livré à la «liberté d'entreprendre», comme le préconise de façon si déterminée Thomas Heiniger, à la tête de la Direction de la santé à Zurich. Les investisseurs privés pourraient alors reprendre l'hôpital et pousser au rendement, pour satisfaire leurs actionnaires. Ils voudront se débarrasser des spécialités (et des patients) les moins rentables. Les cantons étant responsables de la sécurité des soins, ils devront recommencer à gérer leurs propres hôpitaux pour les patients âgés, ceux qui présentent des pathologies multiples ou marginales, et ceux souffrant de dépendances ou de handicaps. A entendre les partisans de l'orthodoxie libérale, on a l'impression qu'un hôpital public n'est pas capable d'agir en fonction de la situation, d'établir des coopérations, ni de se procurer du capital. C'est absurde. Tout dépend du contexte légal. Autre argument des dérégulateurs: si les cantons à la fois gèrent, financent et contrôlent les hôpitaux, cela risque d'entraîner des conflits de rôles. Mais il faudrait alors aussi livrer à la «liberté d'entreprendre» les écoles des niveaux primaires et secondaires, les écoles professionnelles et les hautes écoles spécialisées. Les plaintes des écoles et des hôpitaux privés se rejoignent, par exemple sur les prétendus préjudices subis lors de l'indemnisation des prestations de service public.

La concurrence ne change rien au fait que le service public doit garantir à tous l'accès aux prestations de santé. Il peut en principe déléguer cette responsabilité à des acteurs privés. Mais il faut alors garantir que les cas les plus délicats ne soient pas refusés et que les fonds issus des taxes et primes ne viennent pas alimenter la participation aux bénéfices des actionnaires. Cela implique donc de nouveau un système de contrôle, afin de garantir le respect des règles. Les études actuelles ne font état d'aucun avantage de la privatisation et de la concurrence dans le paysage hospitalier, que ce soit aux Etats-Unis ou ailleurs. Les résultats des études correspondantes sont parfois contradictoires, ou vont dans le sens contraire. Un article de la littérature allemande daté de 2012 arrive par exemple à la conclusion, surprenante pour les auteurs, que «les gestionnaires privés des cliniques ne sont pas globalement plus efficaces, et que les hôpitaux publics et d'utilité publique ne sont pas globalement moins efficaces.» Pour savoir quelle est la meilleure forme d'organisation pour les hôpitaux, il faut se fier à ce que l'on constate actuellement: les hôpitaux sont plus ou moins bien gérés, indépendamment du fait qu'ils soient publics ou privés, d'utilité publique ou orientés vers le profit.

\section{Références}

1 La vie économique 3/2017.

2 Sibbel R, Nagarajah B. Sind die privaten Krankenhausträger effizienter? - Ergebnisse aus der internationalen Literatur (en allemand / le financement privé des hôpitaux est-il plus efficace? - résultats de la littérature internationale). Gesundheitswesen. 2012;74:379-86. 